\section{Fast luminance measurement method for asynchronous spiking pixels}

\section{Juan A. Leñero-Bardallo and Francisco J. García-Pacheco}

We present a new method to obtain a continuous and fast measurement of light intensity. It is targeted for Integrate and Fire (I\&F) pixels that pulse with a frequency proportional to illumination. The procedure is intended to speed up the pixel readout of low illuminated pixels. It does not require synchronization of different digital signals, being compatible with continuous pixel operation. The method fundamentals are described in the letter. Experimental results validating the theory are provided.

Introduction: Luminance sensors with asynchronous spiking pixels are made up of pixels that pulse with a frequency proportional to illumination $[1,2,3]$. They have several inherent advantages over frame-based sensors: 1) Pixels operate continuously and there in not necessary to set an integration time; 2) Under average illumination conditions, pixel latency and power consumption are low; 3) Non illuminated pixels do not send any data off-chip, optimizing the bandwidth consumption. For these reasons, such sensors are suitable for specific applications that require good temporal resolution [4] or avoid reading out dark pixels [5]

In Fig. 1, the operation principle of an Integrate and Fire (I\&F) pixel is depicted. Initially, the voltage at the integration capacitance, $V_{c}$ is reset. When the reset signal is disabled, the capacitor is discharged with a slope that is proportional to illumination. If the voltage threshold $V_{\text {ref }}$ is reached, the pixel sends out a pulse and thereafter self-resets. It can be easily demonstrated that the spiking frequency is given by:

$$
f_{o s c} \approx \frac{I_{p h}}{C \cdot\left(V_{D D}-V_{r e f}\right)}=\frac{I_{p h}}{C \cdot \Delta V}
$$

Where $V_{\text {reset }} \approx V_{D D}$ and $\Delta V=V_{D D}-V_{\text {ref }}$. The integration capacitance is dimensioned to obtain spiking frequencies in the order of milliseconds under average indoor conditions $[6,3]$. Illumination levels are rendered by gauging the output frequency associated to each pixel.

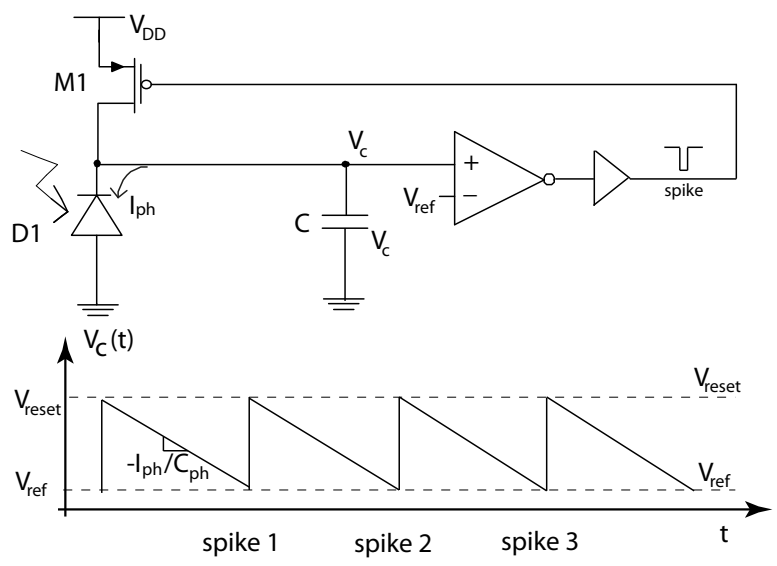

Fig. 1 Top: Integrate \& Fire pixel block diagram excluding the readout circuitry. Bottom: Transient voltage at the integration capacitance.

Spiking sensors main disadvantage is slow operation under low illumination conditions. Since pixels spiking frequencies are proportional to illumination, pixel response can be very slow. That limits the sensor performance in low illuminated environments. To overcome this drawback, it is possible to increase globally the sensor sensitivity to light by decreasing the comparator's voltage threshold, $\Delta V$ in Eq. 1, tuning the $V_{r e f}$ voltage. Low $\Delta V$ values are suitable for low illuminated pixels. However, this traditional procedure has a negative impact with high illuminated pixels: increases their spiking rate, fixed-pattern-noise (FPN) and power consumption unnecessarily. Ideally, low values of $\Delta V$ should be set during a short time interval to measure the low illuminated regions with an acceptable latency.

We propose an alternative technique to speed up the pixel readout of low illuminated pixels without increasing significantly the sensor's throughput, FPN and power consumption. The approach allows the measurement of pixel illumination values with a maximum latency time that can be set by the user and can be adapted dynamically depending on the minimum illumination value and the maximum output data flow required. Low values of $\Delta V$ are set during a short time interval just to speed up the response of low illuminated pixels.

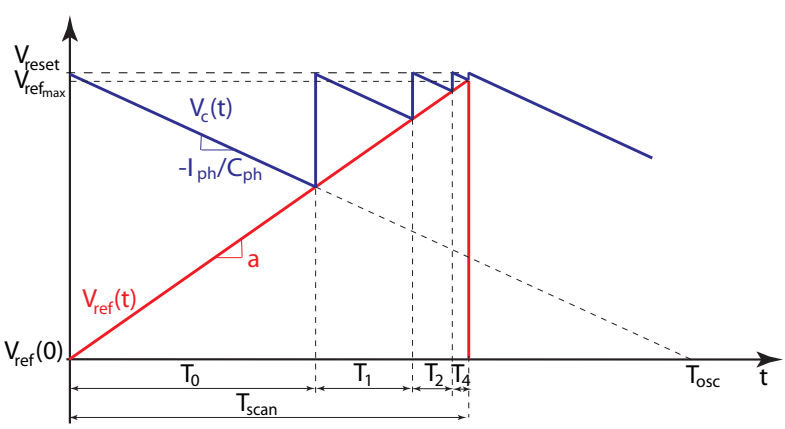

Fig. 2 Proposed pixel readout method for low illuminated pixels. The comparator's threshold voltage is sweep between its initial value, $V_{\text {ref }}(0)$, and a final one, $V_{\text {ref } f_{\text {max }}}$, within a time interval, $T_{\text {scan }}$. As a result, pixel outputs can be readout earlier than keeping the initial voltage threshold constant.

Method description: Initially the pixel $V_{\text {ref }}$ voltage is set to $V_{\text {ref }}(0)=$ $V_{r e f_{0}}$. We aim to speed up the pixel response in low illuminated regions without setting a constant low $\Delta V$ value. To do so, we propose to change the comparator's threshold signal $V_{r e f}$ dynamically, as is depicted in Fig. 2. Keeping the initial voltage threshold constant, would require an amount of time equal to $T_{\text {scan }}$ for the pixel readout. To expedite the pixel response, the signal that represents the transient voltage at the integration capacitance, $V_{c}(t)$, is modulated by the signal $V_{\text {ref }}(t)=a \cdot t+V_{\text {ref }}(0)$.

Let us denote as $T_{0}, T_{1}, T_{2}, \ldots, T_{n}$ the different temporal values where $V_{c}\left(T_{i}\right)=V_{\text {ref }}\left(T_{i}\right)$, with $i=0,1, \ldots, n$, and the pixel fires. Our target is to determine them as a function of the photocurrent and the slope of the signal $V_{\text {ref }}(t)$. Assuming that $V_{c}(0)=V_{D D}$ and $V_{\text {ref }}(0)=V_{\text {re }}$, we can express both signals as follows:

$$
\begin{aligned}
V_{c}(t) & =V_{D D}-\frac{I_{p h} \cdot t}{C} \\
V_{\text {ref }}(t) & =a \cdot t+V_{\text {ref }}
\end{aligned}
$$

Using Equations 2 and 3, the first spike timestamp, $T_{0}$, and the voltage value $V_{c}\left(T_{0}\right)=V_{\text {ref }}\left(T_{0}\right)$ can be determined:

$$
\begin{aligned}
T_{0} & =\frac{V_{D D}-V_{r e f_{0}}}{a+\frac{I_{p h}}{C}} \\
V_{\text {ref }}\left(T_{0}\right) & =a \cdot T_{0}+V_{\text {ref }}
\end{aligned}
$$

Again, with Equations 2 and 3, and the values of $T_{0}$ and $V_{\text {ref }}\left(T_{0}\right), T_{1}$ and $V_{r e f}\left(T_{1}\right)$ can be computed:

$$
\begin{gathered}
T_{1}=\frac{V_{D D}-V_{\text {ref }}\left(T_{0}\right)}{a+\frac{I_{p h}}{C}} \\
V_{\text {ref }}\left(T_{1}\right)=a \cdot\left(T_{0}+T_{1}\right)+V_{\text {ref }}
\end{gathered}
$$

Therefore, from the previous equations we can generalize and express $V_{\text {ref }}\left(T_{n}\right)$ and $T_{n}$, with $n>1$, as follows:

$$
\begin{gathered}
V_{\text {ref }}\left(T_{n}\right)=a \cdot\left(T_{0}+T_{1}+\ldots+T_{n-1}\right)+V_{\text {ref }} \\
T_{n}=\frac{V_{D D}-V_{\text {ref }}\left(T_{n-1}\right)}{a+\frac{I p h}{C}}= \\
=T_{0}-\frac{a\left(T_{0}+T_{1}+\ldots+T_{n-1}\right)}{a+\frac{I_{p h}}{C}}= \\
=T_{0}-A \cdot\left(T_{0}+T_{1}+\ldots+T_{n-1}\right)
\end{gathered}
$$

With $A=\frac{a}{a+I_{p h} / C}$. From Equation 8, a general expression of $T_{n}$ as a function of $T_{0}$ can be derived: 


$$
\begin{gathered}
T_{0}=T_{0} \\
T_{1}=T_{0}-A T_{0}=(1-A) T_{0} \\
T_{2}=T_{0}-A\left(T_{0}+T_{1}\right)=(1-A)^{2} T_{0} \\
\vdots=\vdots \\
T_{n}=(1-A)^{n} \cdot T_{0}
\end{gathered}
$$

Hence, from Equation 9, we can state that the ratio between two consecutive time intervals is always constant and depends on $I_{p h}$ :

$$
\frac{T_{n}}{T_{n-1}}=(1-A)
$$

The proposed method is mainly targeted for a fast measurement of low illuminated regions. Hence, for these pixels the slope of $V_{r e f}(t)$ will be much higher than the slope of $V_{c}(t)$, i.e. $a>>I_{p h} / C$. Thus, the ratio between two consecutive time intervals is approximately:

$$
\begin{array}{r}
A=\frac{a}{a+I_{p h} / C} \approx 1-\frac{I_{p h}}{a \cdot C} \\
\frac{T_{n}}{T_{n-1}}=(1-A) \approx \frac{I_{p h}}{a \cdot C}
\end{array}
$$

Taking into account the amount of time that lasts between two consecutive events fired by one pixel after $t=0$, it is possible to measure the illumination value within a reduced time interval, $T_{\text {scan }}$, set by the user:

$$
T_{\text {scan }}=\frac{V_{r e f_{\max }}-V_{r e f_{0}}}{a}
$$

In case the pixel illumination is not high enough to generate at least two events during $T_{\text {scan }}$, the pixel can be considered to be dark. The user can trade off between the number of events fired by the pixels and $T_{\text {scan }}$.

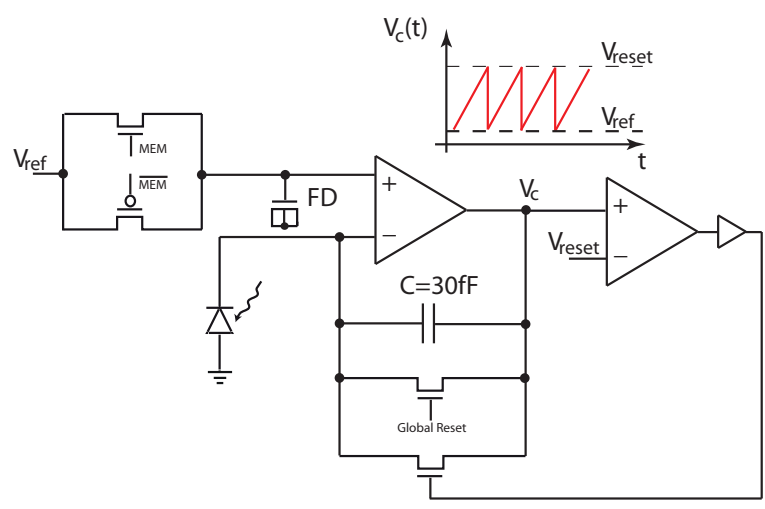

Fig. 3 Integrated I\&F pixel schematics. Digital readout circuitry is excluded. Voltages $V_{\text {ref }}$ and $V_{\text {reset }}$ can be tuned independently. There is a floating diffusion (FD) to store the value of $V_{\text {re } f_{0}}$ on pixel.

Experimental results: Fig. 3 displays the schematics of an I\&F pixel integrated in the AMS HV 180nm standard fabrication technology. Digital readout logic is not shown. The pixel design allows full programmability of the voltages $V_{\text {ref }}$ and $V_{\text {reset }}$. A floating diffusion was included to store locally on each pixel a different value of $V_{r e f_{0}}$. The memory should refreshed periodically. Measurements last much less than the refresh time. Pixel operation is very similar to the described in Fig. 1, but in this implementation, the slope of the $V_{c}(t)$ signal is positive.

In Fig. 4 experimental results measured with the implemented pixel are shown. The pixel was illuminated with uniform constant illumination. Voltages $V_{c}, V_{\text {ref }}$ and the pulses fired by the pixel where recorded with an oscilloscope. In the top plot, we display the transient voltage $V_{\text {ref }}(t)$ and the voltage at the integration capacitance, $V_{c}(t)$. In the middle plot, the timestamps of each pulse fired by the pixel are displayed. It can be noticed how the time interval between consecutive pulses depends on the value of $V_{\text {ref }}(t)$. On the bottom plot, the ratio between time intervals is plotted. As it is predicted by Equation 12, it is approximately constant. The error and the spiking frequency are higher when $V_{r e f}(t)$ rises. It should be noticed that when $V_{r e f}(t)$ is decreasing the ratio between consecutive periods predicted by Equation 12 is inverted, i.e. $T_{n} / T_{n-1} \approx$ $\frac{a \cdot C}{I_{p h}}$. Averaging different measured values of $T_{n} / T_{n-1}$, measurement errors can be significantly minimized.
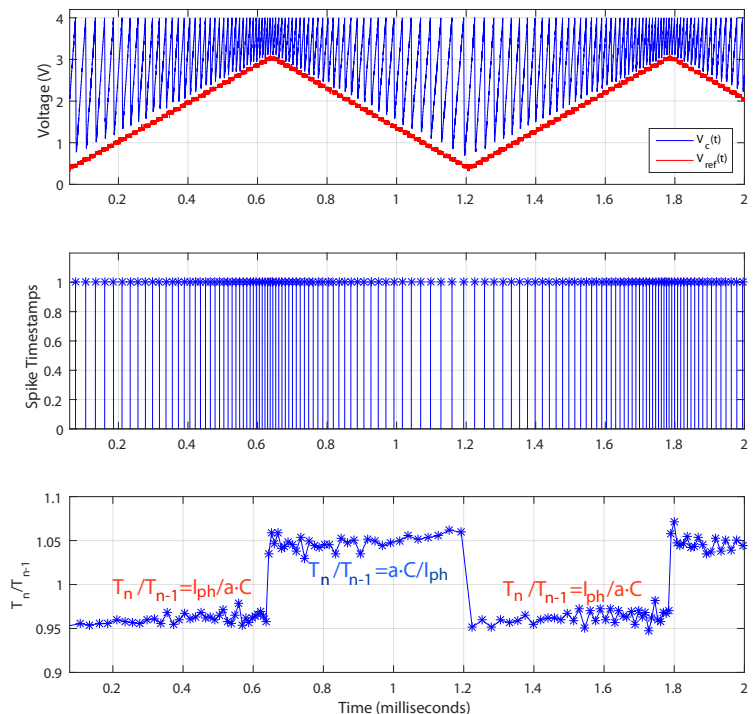

Fig. 4 Top: Measured transient voltages $V_{c}(t)$ and $V_{\text {ref }}(t)$. Center: Recorded pixel spike timestamps, $T_{n}$. Bottom: Ratio between each measured time interval and the previous one $\left(T_{n} / T_{n-1}\right)$. As it is predicted by Equation 12, the ratio is constant for a given illumination value.

Conclusion: A new procedure to speed up the measurement of the pixel illumination for I\&F spiking pixels has been described and demonstrated. To accelerate the pixel response, we have proposed to change dynamically the pixel sensitivity to light varying the value of a pixel comparator threshold. Just two time intervals between consecutive spikes are necessary to measure pixel illumination. Lower illumination levels can be measured at the expense of increasing temporarily the pixel throughput. We intend to employ this method to readout pixels of low illuminated regions. To do so, circuitry to define an specific value for the voltage threshold within a certain group of pixels of a pixel array is being developed.

Acknowledgment: This work has been supported in part by the University of Cádiz under Grant PR2016-072; in part by the Spanish Ministry of Economy and Competitiveness under Grant TEC201566878-C3-1-R, Co-Funded by ERDF-FEDER; and in part by Junta de Andalucía CEICE under Grant TIC 2012-2338 (SMARTCIS-3D).

Juan A. Leñero-Bardallo and Francisco J. García-Pacheco (University of Cádiz, Spain)

E-mail: juanantonio.lenero@uca.es

\section{References}

1 E. Culurciello, R. Etienne-Cummings, and K. Boahen, "A biomorphic digital image sensor," Solid-State Circuits, IEEE Journal of, vol. 38, no. 2, pp. 281-294, Feb 2003.

2 M. Dei et al., "Highly linear integrate-and-fire modulators with soft reset for low-power high-speed imagers," in Proceedings of the 2017 IEEE International Symposium on Circuits and Systems, ISCAS 2017, Baltimore, USA, June 2017, pp. 1-4.

3 J. A. Leñero-Bardallo, R. Carmona-Galán, and A. Rodríguez-Vázquez, "A wide linear dynamic range image sensor based on asynchronous self-reset and tagging of saturation events," IEEE Journal of Solid-State Circuits, vol. 52, no. 6, pp. 1605-1617, April 2017.

4 J. Leñero-Bardallo, D. Bryn, and P. Häfliger, "Flame monitoring with an AER color vision sensor," in Circuits and Systems (ISCAS), 2013 IEEE International Symposium on, May 2013, pp. 2404-2407.

5 J. A. Leñero-Bardallo, L. Farian, J. M. Guerrero-Rodríguez, R. CarmonaGalán, and . Rodríguez-Vázquez, "Sun sensor based on a luminance spiking pixel array," IEEE Sensors Journal, vol. 17, no. 20, pp. 6578-6588, Oct 2017.

6 J. A. Leñero-Bardallo, D. Bryn, and P. Häfliger, "Bio-inspired asynchronous pixel event tricolor vision sensor," Biomedical Circuits and Systems, IEEE Transactions on, vol. 8, no. 3, pp. 345-357, June 2014. 